\title{
Bisphosphonate related osteonecrosis of the jaw in
}

\section{non-malignant bone disease}

Peter KK Wong ${ }^{1}$

Gelsomina L Borromeo ${ }^{2}$

John D Wark ${ }^{3}$

1 Mid-North Coast Arthritis Clinic, Coffs Harbour; and Rural Clinical School, Faculty of Medicine, University of New South Wales, New South Wales, Australia.

2 Melbourne Dental School, The University of Melbourne, Parkville, Melbourne, Victoria, Australia.

3 Department of Medicine, University of Melbourne and Bone and Mineral Medicine, Royal Melbourne Hospital, Parkville, Melbourne, Victoria, Australia. 


\section{Corresponding Author:}

A/Professor Peter Wong

Mid-North Coast Arthritis Clinic

PO Box 6307

Coffs Harbour

New South Wales 2450

\section{AUSTRALIA}

Phone: +61-2- 66529822

Fax: +61-2- 66516177

Email: peter.wong@westnet.com.au

Key words: bisphosphonate, osteonecrosis, jaw, non-malignant, bone

Word Count: 4066 


\begin{abstract}
Bisphosphonates such as alendronate, risedronate and zoledronate have revolutionised the treatment of osteoporosis and Paget's disease. These drugs reduce fracture risk and probably even mortality in patients with osteoporosis. However, they have a long in vivo half-life following cessation and may be associated with delayed dental healing and even the devastating complication of osteonecrosis of the jaw (ONJ). Extensive media attention highlighting this issue has caused much concern among patients and health care professionals. This paper seeks to provide treating clinicians with a balanced multidisciplinary review of the available evidence pertaining to this issue and practical advice regarding prevention and management of ONJ.
\end{abstract}




\section{Bisphosphonates in non-malignant bone disease}

Osteoporotic fractures are a major public health problem in most Western countries. Patients sustaining a hip fracture have a $33 \%$ mortality rate in the following 12 months $[1,2]$. This excess mortality may persist for up to 10 years following fracture $[3,4]$. Up to one third of such patients subsequently require admission to residential care [5].

Bisphosphonates (BPs) such as alendronate (ALN), risedronate (RSD), ibandronate and zoledronate (ZLD) are first-line treatments for osteoporosis, especially following low-trauma fracture [6]. In Australia (population 22.5 million people), 1.3 million BP scripts were written for the treatment of osteoporosis in the 12 months from July 2009 to June 2010. This is similar to the number of prescriptions for celecoxib, a cyclo-oxygenase-2 inhibitor used for symptom relief in arthritis and budesonide, a common inhaled corticosteroid used for asthma [7]. Paget's disease is another common benign bone disorder for which BPs are indicated [8]. 
Annual intravenous (IV) ZLD in post-menopausal women was well-tolerated and increased bone mineral density (BMD) by up to $5 \%$ at 12 months and lowered the risk of vertebral and hip fracture over three years $[10,11]$. An additional three years of ZLD maintained the initial BMD gain [12]. Zoledronate administration within 90 days following surgical repair of a fractured hip reduced the risk of another fracture and improved patient survival - possibly due to a beneficial effect on cardiovascular events and pneumonia [13-14]. The decline in hip fracture incidence in the US occurred in parallel with increased BP use [15]. Australian data suggested oral BPs were associated with a lower mortality rate in women and men compared to those on no treatment [16].

A succession of case reports since 2003 highlighted the possible association between BPs, especially ZLD or pamidronate (APD), and ONJ [17-23]. However, the American Society of Bone and Mineral Research (ASBMR) noted the quality of reported evidence was often poor [24]. Incomplete patient data, lack of prospective patient follow-up and incomplete reporting of BP parameters made accurate estimation of ONJ prevalence difficult [25,26]. ONJ has been the subject of much media attention, capturing public imagination and resulting in atrisk patients refusing BPs [27]. This paper seeks to provide treating clinicians with a balanced multi-disciplinary review of the available evidence pertaining to this issue and practical advice regarding prevention and management of ONJ.

\section{$\underline{\text { Methods }}$}

We identified published studies by performing a computerized literature search of the PubMed database using the key words 'bisphosphonate' and 'dental' or "jaw" for 
English language papers published during the last 10 years. All key articles were retrieved with articles in all journals considered. Reference lists of published papers were explored to identify additional relevant papers. The authors independently reviewed and judged the selected publications on their contribution to knowledge on the topic then reached a consensus on which publications to include in this review. As previously noted by the ASBMR the quality of reported evidence was often poor [24]. All papers were Level III or IV evidence - case control studies or case series, respectively.

\section{Diagnosis of ONJ}

The American Association of Oral and Maxillofacial Surgeons (AAOMS) proposed a definition of ONJ as "exposed necrotic bone in the maxillofacial region that has persisted for more than 8 weeks" in the absence of a history of radiation therapy to the jaw (Figure 1) $[28,29]$. The ASBMR and a European Working Group also suggested an 8-week cut-off as trauma, extractions and oral surgical procedures are usually followed by tissue healing within this time [24,30]. The ASBMR guidelines highlighted several important differential diagnoses including periodontal disease, gingivitis, mucositis, osteomyelitis, sinusitis, temporo-mandibular joint disease, osteoradionecrosis and bone tumours [24]. The possible features of BP-related ONJ (BRONJ) are outlined in Table 1 [24,31-34]. However, as none of these are specific for ONJ a careful medical and dental history is crucial - especially the dose/duration of BP therapy and presence of preceding dental trauma. Radiographs are essential to exclude alternative diagnoses such as malignancy [34]. 


\section{Pathogenesis of ONJ}

BPs are preferentially deposited in bone where they inhibit osteoclast activation and promote osteoclast apoptosis by several mechanisms including inhibition of protein prenylation and blockade of mevalonate metabolism [35-37]. BPs appear anti-angiogenic [38-40] and may inhibit matrix metalloproteinases (the enzymes involved in tumour metastasis and connective tissue remodelling) [41,42]; and in vitro proliferation of oral keratinocytes [41]. Once taken up by bone, BPs are usually eliminated during bone resorption - a process taking some years [43]. This results in a long bone half-life, for example up to 10 years for ALN [44]. Differences in binding affinities of the various BPs for hydroxyapatite affect release from bone and may explain the observed clinical differences in potency, pharmacokinetics and duration of effect [45].

Mucosal damage following extraction, irritation from ill-fitting dentures or insertion of dental implants complicated by infection and bone necrosis in the setting of BP-induced low bone turnover may contribute to ONJ. BPs may exert an inhibitory effect on oral epithelial growth [46]. In vitro work using human gingival fibroblast and keratinocyte cell lines found ZLD inhibited cellular proliferation and promoted apoptosis [47]. These findings may explain the upper gastro-intestinal side effects seen with orally administered BPs and the oral ulceration described following chewing of BP tablets [46,48]. Various disease states such as diabetes, renal failure or immunosuppression may impair tissue healing and result in infection. Due to their highly polar nature, oral BPs are poorly absorbed from the gastrointestinal tract and therefore have low bioavailability - often less than $2 \%[49,50]$. Oral BPs also have lower potency, for example ALN and RSD are approximately 30-50 times less potent than ZLD [51]. The higher bioavailability and potency of IV BPs may explain their 
stronger association with ONJ. However, it is for these very reasons and the convenient mode of administration that IV BPs are used.

Detailed histomorphometric analysis of jaw bone samples from patients with probable BRONJ (26/31 with an underlying malignancy) revealed little osteoclastic activity or new bone formation compared to corresponding samples from controls [32]. Bone obtained from cases appeared thicker and less vascular - especially in the setting of infection.

While osteonecrosis may occur at other anatomic sites, for example the femoral head or femoral condyle, these sites are sterile and therefore do not progress to persistent frank bone necrosis. In contrast, the mouth is a unique anatomic site because abundant micro-organisms have ready access to bone $[23,52]$. Furthermore, jaw bones undergo repeated trauma from masticatory activity [53] and have an extensive blood supply allowing BPs to achieve high local concentrations $[54,55]$.

The central role of osteoclast inhibition in the pathogenesis of ONJ is supported by recent case reports of a similar complication in patients receiving denosumab, a monoclonal antibody blocking Receptor Activator of Nuclear Factor kappa-B Ligand (RANKL), a crucial cytokine promoting osteoclastogenesis [56,57]. Most of these cases have been described in patients with an underlying malignancy receiving large doses of denosumab $(120 \mathrm{mg} \mathrm{s} / \mathrm{c}$ every 4 weeks). No cases of ONJ were reported in the pivotal FREEDOM study of 7868 women with PMO treated with denosumab (60 mg s/c every 6 months) for three years [58]. Two cases of ONJ, both of which subsequently healed, were reported in the five year study extension [59]. 


\section{Animal models of ONJ}

Development of a suitable animal model would assist clarification of the pathogenesis and treatment of ONJ. Several rat models of BRONJ have been reported [60-63]. Extraction of an upper molar tooth was performed in Wistar rats following weekly administration of ZLD (0.04 mg IV weekly for five weeks). Seven weeks post-extraction all rats treated with ZLD showed expansion of the surgical defect and exposed bone. Irregularity of the cortical margin and bone destruction was noted while histologic analysis of the defect revealed necrotic bone with osteocyte loss [60]. Another study involving rats compared the risk of jaw necrosis post-dental extraction following administration of ALN (0.05 mg/kg weekly by gavage) or ZLD (0.6 mg/kg intra-peritoneally weekly). Eighty percent of rats given ZLD had histologic osteonecrosis while no changes were seen following ALN or in controls [61]. Rats administered ZLD at a dose of $7.5 \mathrm{mcg} / \mathrm{kg}$ once a week for up to three weeks and dexamethasone prior to molar extraction developed ulceration with underlying areas of necrotic bone [62]. However, the above ZLD regimes were more analogous to the large doses seen in oncology rather than the smaller doses used for non-malignant bone disease [60-62].

Others have suggested a canine model of ONJ may be more appropriate as canine bone turnover is more similar to that seen in humans $[64,65]$. The recent development of murine models of BRONJ is exciting as this will allow access to the extensive experimental tools and well-defined immunology of genetically altered mice $[66,67]$. However, caution needs to be exercised in extrapolating data from animal studies to humans as there are considerable differences in bone re-modelling and oral function.

\section{Bisphosphonates and osteonecrosis of the jaw (ONJ)}


Previously, clinically similar but less frequent presentations occurred following radiotherapy (Figure 2) or occupational exposure to phosphorus $[19,68]$. ONJ appears mainly in the setting of malignancy, especially multiple myeloma [69-71] or breast cancer [20-22,72] - possibly due to the greater cumulative doses of BP used to treat these cancers [73]. In myeloma patients, IV ZLD and APD were associated with a $10 \%$ and $3 \%$ incidence of ONJ at 36 months, respectively [70]. A longitudinal cohort study examined 1621 patients who received 29006 doses of IV BP and found the crude incidence of ONJ was $8.5 \%, 3.1 \%$, and $4.9 \%$ in patients with myeloma, breast cancer and prostate cancer, respectively [74]. However, a retrospective study of 2239 cancer patients suggested a lower prevalence (1.3\%) of BRONJ [73]. A systematic review identified 99 cases of ONJ in patients treated with a BP for nonmalignant bone disease [75]. Eighty-nine percent of cases with a recorded dental history underwent a preceding dental procedure; $71 \%$ of cases were taking at least one bone-active medication (usually a corticosteroid) in addition to the BP while $81 \%$ of patients had additional co-morbid conditions such as rheumatoid arthritis and diabetes predisposing to poor wound healing [75].

Review of 119 patients with probable BRONJ suggested the mean time to clinical presentation was 14.3 months for APD, 9.4 months for ZLD and three years for those receiving ALN [54]. Almost all these patients were treated with IV BPs for malignant bone disease and ONJ appeared to be precipitated by dental trauma, for example tooth extraction or ill-fitting dentures [17,19-21]. Co-existent periodontitis, a bacterial plaque-related disease resulting in gingival inflammation and alveolar bone resorption was often noted [54]. However, potential confounders, for example chemotherapy, radiotherapy, corticosteroid use and dental/sinus infections complicated attribution of causality [76]. A retrospective analysis of 4019 cancer patients treated with IV BPs identified high cumulative BP dose, poor oral 
health and dental extraction as risk factors for ONJ [73] while others have suggested a possible association with diabetes [77].

\section{Bisphosphonates and ONJ in non-malignant bone disease}

There appear markedly fewer cases of ONJ during treatment with BPs for osteoporosis $[19,22,54,78,79]$. However, a recent study reported 135 consecutive patients from June 2005 to May 2009 with probable BRONJ [80]. Twenty-five of these patients were treated for osteoporosis with a mean duration of BP therapy of 50 months [80]. Review of patients at a single academic dental centre identified 208 patients with a history of ALN use [81]. Of these, nine (4\%) had ONJ - all following tooth extraction or other dental trauma resulting in bone exposure. In comparison, of the 13522 patients at the same centre without a recorded history of ALN use, 4384 (32\%) underwent dental extraction with no reported ONJ. A retrospective study from 11 different European centres reported 47 cases of probable BRONJ diagnosed between 2004 and 2008 [82]. Eight percent (37/470) of cases appeared to be associated with BP therapy for osteoporosis with a mean duration of oral BP use of 57.85 .3 months [82].

A study of patients with post-menopausal osteoporosis (PMO) receiving ALN for up to 10 years reported no cases of ONJ [83]. No BRONJ cases were reported from the risedronate and alendronate clinical trials (each involving16,000-17,000 patients) [84]. $\underline{\text { A systematic literature review found incidence rates for BRONJ among patients with }}$ OSP varied from $0.028 \%$ to $4.3 \%$ [85]. A nested case control study within a cohort of $\underline{\text { six million individuals aged }>55 \text { years from an Italian claims database found an }}$ adjusted OR for ONJ in current BP users was 2.8 (95\% CI 1.3-5.9) against never users 
[86]. The American Dental Association (ADA) concluded the risk of ONJ in patients taking oral BPs was considerably less compared to that seen following IV BP for malignancy [87]. A double blind placebo controlled trial of 3889 patients with PMO who received three infusions of ZLD over a two-year period reported no ONJ cases [11]. Blinded adjudication of the database subsequently yielded one case each of delayed dental healing in the ZLD and control groups. Both cases occurred following dental manipulation and resolved with antibiotics and debridement [11]. No further ONJ cases were reported in the three year extension of this study nor following review of data from four other clinical trials involving patients with low BMD treated with ZLD $[12,88]$.

While there may be less published evidence that chronic low-dose BP therapy for osteoporosis or other non-malignant bone disease is associated with $\mathrm{ONJ}$, this may change as more people are treated for longer periods of time with these agents. The lack of ONJ cases reported from the alendronate and risedronate clinical trial programmes suggest a low frequency of this complication. There may also be a long latency of onset due to the relatively low doses of oral BP administered during the trial. Furthermore delayed dental healing/ONJ was not one of the secondary outcomes specifically captured during these trials. Patients with non-malignant bone disease usually live longer than those with an underlying malignancy, making them candidates for a drug-related complication with a long latency of onset. As BPs have a long in vivo half-life of up to a decade $[89,90]$ and ALN suppressed bone turnover markers for up to five years following cessation [83], it is possible that increasing numbers of patients will present with delayed dental healing over the next few years. It is difficult to extrapolate findings from the oncologic literature to non-malignant bone disease as BP doses for malignant bone disease are up to ten-fold higher than those used in osteoporosis, for example, ZLD $4 \mathrm{mg}$ IV 4 weekly [90] and ZLD $4 \mathrm{mg}$ IV annually [10], 
respectively. The exact risk of $\mathrm{ONJ}$ in non-malignant bone disease is uncertain. Australian data acquired via postal survey of oral and maxillofacial surgeons suggested a risk as high as 1:2260-1:8460 in patients taking weekly ALN for osteoporosis [91]. However, the retrospective study design and lack of adjudicated diagnosis may have resulted in referral/recall bias and uncertain case definition - thus elevating the risk. The ASMBR suggested a more conservative risk estimate of 1:10 000-1:100 000 for patients with osteoporosis taking BPs [24].

A retrospective study using data from two Health Maintenance Organizations (HMO) attempted to assess the incidence of ONJ and estimate odd ratios (OR) for ONJ risk following BP exposure [92]. Twenty three cases of ONJ were identified from 572606 members with a calculated incidence of 0.63 (95\%CI: 0.39-1.59) per 100000 person years. However, study limitations included the quality of data entered in the electronic medical record, lack of a specific diagnostic code for ONJ and recall bias by treating clinicians. With these caveats the OR $(95 \% \mathrm{CI})$ of ONJ associated with the following risk factors were as follows: oral BP use 9.2 (3.6-23.3), head/neck radiation 14.2 (4.8-41.6), any cancer 7.6 (3.417.3), corticosteroids $3.0(1.3-6.8)$ and diabetes $1.6(0.5-4.6)$ [59]. A case control study ( $n=308$ cases) involving patients from US community-based dental practices found that BP use for more than two years, oral suppuration and dental extractions were independent risk factors for ONJ [93]. In cases with non-malignant bone disease, BP use less than two years, two to five years and more than five years had OR (95\%CI) of 5.2 (1.2-22.5), 11.4 (3.2-40.2), and 26.6 (5.3-133.6), respectively [93]. In another study $13946 \mathrm{HMO}$ members taking oral BPs were contacted by mail. Of the 8572 respondents, 2159 reported relevant dental symptoms. Of these, 1005 agreed to a dental examination and nine cases of ONJ were identified, yielding a prevalence of $0.10 \%$ [94]. 
$\underline{\text { A retrospective cohort study of } 30 \text { cases of BRONJ all of whom had received oral BP }}$ for osteoporosis found that healing was slower in those developing BRONJ following dental extraction especially in the presence of co-morbidities such as rheumatoid arthritis, diabetes, glucocorticoid therapy, and disease-modifying anti-rheumatic drugs [95].

A case control study to assess the risk of ONJ in Australian patients receiving BP for nonmalignant bone disease has been performed [96]. The primary outcome was the incidence of delayed dental healing occurring either spontaneously or following dental procedures [96]. Study results will assist in clarifying the risk of delayed dental healing following BP use in patients with non-malignant bone disease and in controls. Unfortunately, the risk of delayed dental healing/exposed bone in the absence of BP use is not definitively known. This has hampered attribution of causality as exposed oral bone in the maxillofacial region which fails to heal despite conservative measures has also been reported in the absence of BP exposure [24,97].

\section{Prevention of ONJ}

Although published guidelines regarding prevention and treatment of ONJ exist, they are based on expert opinion rather than firm data [28,87,98-100]. While there remains much uncertainty among the dental, oral surgical and medical professions regarding optimal dental management of patients prior to and during BP therapy these guidelines have encouraged dialogue between specialties. A staging system developed by AAOMS should assist classification of severity and standardisation of communication (Table 2) [29,55]. 
It seems sensible to arrange dental review prior to BP commencement so any invasive dental procedures can be performed $[29,34,53]$. The ADA suggest routine dental care should not be modified solely because of coexistent oral BP use [87]. Dentures should be checked to ensure they are well-fitting and areas of denture trauma identified. This is a good opportunity to educate patients regarding dental hygiene and the need for regular dental care as they are often poorly informed about the reason for BP therapy and the possible impact on dental health [101]. Patients should be instructed to promptly report any new dental symptoms especially dental pain, swelling or exposed bone. During BP therapy, active early treatment of dental infection should be undertaken and invasive dental therapy performed only if necessary, and in consultation with the treating medical practitioner. Implementation of such preventive measures and delaying ZLD administration for 6-8 weeks following invasive dental procedures to allow wound healing reduced the risk of ONJ in oncological patients $[102,103]$. No data suggest BP cessation prior to invasive dental surgery influences outcome $[23,30]$. This should be determined on a case-by-case basis upon an assessment of current fracture risk and following consultation between members of the treating team [104]. The prolonged suppression of bone turnover following cessation of ALN [83] means stopping it for even months prior to any invasive dental procedure may not have a meaningful biologic effect. In contrast the shorter in vivo half-life of RSD suggests its cessation for some months prior to any invasive dental procedure may be appropriate [51].

$\underline{\text { A recent prospective study involving } 700 \text { consecutive patients taking oral BPs for OSP }}$ who underwent a total of 1480 dental extractions found that antibiotic coverage in combination with either delicate surgery and closure by primary intention or non- 
$\underline{\text { traumatic avulsion and closure by secondary intention resulted in no cases of BRONJ }}$

[105].

An uncontrolled retrospective study in patients with osteoporosis suggested a greater risk of ONJ in those with a more suppressed serum C-telopeptide (CTx), a marker of bone resorption [105]. However, the level of serum CTx identified in this paper as "high risk" for development of ONJ is seen in many patients after three years of ALN [106]. Furthermore, the presence of a low serum CTx following annual IV ZLD in patients with PMO was not associated with a high risk of ONJ $[11,88]$. Other work has failed to validate the utility of CTx as a useful predictive biomarker of ONJ [107]. Overall, the evidence for a strong correlation of ONJ risk with serum CTx levels is dubious and the ASBMR has advised this biomarker is not useful in assessing ONJ risk [108].

\section{Management of ONJ}

Once ONJ has developed, therapy involving local debridement, irrigation and antibiotics is usually unsuccessful due to difficulty obtaining an acceptable surgical margin manifested by viable bleeding bone $[54,109]$. Surgical debridement often contributes to tissue breakdown resulting in fistulae and morbidity including halitosis and pain [54,109]. Conservative therapy is preferable with strict control of infection using anti-microbial rinses or systemic antibiotics [24]. However, the outcome of ONJ in patients with benign compared to malignant bone disease may be better due to differences in type, dose and route of BP $[33,79,110]$. Where indicated, necrotic bone and sharp bone edges should be carefully removed under antibiotic cover with minimal tissue/bone trauma. Unlike osteoradionecrosis 
of the jaw, and despite the presence of some case reports suggesting benefit there is no clear evidence that hyperbaric oxygen promotes wound healing $[24,29,111,112]$.

A case report described the successful use of teriparatide (recombinant human parathyroid hormone), a potent bone anabolic agent in promoting healing of the osseous defect in an elderly lady with ALN-related ONJ [113]. The stimulatory effect of teriparatide on bone remodelling may have been responsible for the observed rapid healing of the persistent mandibular bony defect. This finding has been reported by others [114-117]. A randomised controlled study of 40 patients with severe chronic periodontitis found that daily teriparatide was associated with faster resolution of dental osseous defects compared to placebo [117]. While these findings require validation in larger prospective controlled studies they raise the possibility that stimulation of bone remodelling by teriparatide may be helpful in the treatment of ONJ.

\section{Relationship of impaired dental healing to ONJ}

While ONJ is a devastating end-stage complication, impaired dental healing may be a less dramatic earlier, poorly-recognised but more common complication of BP use. Small case series have suggested early BRONJ may present without exposed bone but with jaw bone pain, sinus tract and gingival swelling [118-119]. A recent case series of 332 patients from five European centres identified 96 patients who presented with the "non-exposed" variant of ONJ [120]. Twenty-six percent of these patients were being treated for osteoporosis with BPs [120]. While these patients would not fulfil the initial AAOMS or ASMBR definition of ONJ due to lack of exposed bone [24,28], they suggest an earlier form of ONJ. AAOMS has since introduced "Stage 0" ONJ - "No clinical evidence of necrotic bone” (Table 2) [29]. Prompt 
detection of impaired dental healing is crucial as this may allow intervention with aggressive antibiotic therapy and/or dental hygiene strategies to prevent progression to frank ONJ.

\section{Conclusion}

BPs have revolutionised the treatment of non-malignant bone disease, especially osteoporosis. They reduce fracture risk and probably mortality in patients with osteoporosis. In comparison, the small risk of ONJ in such patients means the benefit:risk ratio is high, especially in those with previous low trauma fracture or a T-score less than -2.5 [121]. While the exact risk of $\mathrm{ONJ}$ in patients using BP for non-malignant bone disease is uncertain the publication of multiple management guidelines has increased awareness of this problem among clinicians. This should translate into timely identification and appropriate early management of new cases of ONJ which may only become apparent over many years due to the long in vivo half-life of BPs and the increasing numbers of patients using these medications.

\section{Key Messages}

Bisphosphonates may be associated with delayed dental healing and osteonecrosis of the jaw 
The risk of dental complications from bisphosphonates at the doses used for nonmalignant bone disease is low. It is much lower than that seen following their use in malignant bone disease. There are several reasons for this including the substantially lower doses of bisphosphonate used in non-malignant bone disease.

This low risk is further minimised by effective communication between the treating dental, surgical and medical health professionals. The risk:benefit ratio of bisphosphonates favours their use for the prevention of osteoporotic fractures.

\section{Conflict of interest}

PKKW has received speaking honoraria, travel support or unrestricted research grants from Amgen, Eli Lilly, Sanofi-Aventis, Novartis and Servier.

JDW has received speaking honoraria, travel support or unrestricted research grants from Amgen, Eli Lilly, GSK, Merck Sharpe and Dohme, Novartis, Sanofi-Aventis and Servier. He has served on advisory boards for Amgen/GSK and Novartis. Specifically, Novartis has provided an unrestricted grant to the authors to conduct a case-control study of delayed dental healing in patients on BPs.

GB has no other competing interests. 


\section{REFERENCES}

1. Jensen JS, Tondevold E (1979) Mortality after hip fractures. Acta Orthop Scand 50(2):1617

2. Keene GS, Parker MJ, Pryor GA (1999) Mortality and morbidity after hip fractures. BMJ 307(6914):1248-50

3. Haentjens P, Magaziner J, Colón-Emeric CS et al (2010) Meta-analysis: excess mortality after hip fracture among older women and men. Ann Intern Med 152(6):380-90

4. Bliuc D, Nguyen ND, Milch VE et al (2009) Mortality risk associated with low-trauma osteoporotic fracture and subsequent fracture in men and women. JAMA 301(5):513-21

5. Marottoli RA, Berkman LF, Leo-Summers L et al (1994) Predictors of mortality and institutionalisation after hip fracture : The New Haven EPESE cohort. Am J Pub Health 84(11):1807-12

6. Sambrook P, Seeman E, Phillips SR et al (2002) Preventing osteoporosis: outcomes of the Australian Fracture Prevention Summit. Med J Aust Apr 15;176:S1-16

7. http://www.pbs.gov.au:80/info/industry/statistics 
8. Reid IR, Miller P, Lyles K et al (2005) Comparison of a single infusion of zoledronic acid with risedronate for Paget's disease. N Engl J Med 353:898-908

\section{Jansen JP, Bergman GJ, Huels J, Olson M (2011) The efficacy of bisphosphonates in} the prevention of vertebral, hip, and nonvertebral-non hip fractures in osteoporosis: a network meta-analysis. Semin Arthritis Rheum 40(4):275-84

10. Reid IR, Brown JP, Burckhardt P et al (2002) Intravenous zoledronic acid in postmenopausal women with low bone mineral density. N Engl J Med 346(9):653-61 11. Black DM, Delmas PD, Eastell R et al (2007) Once-yearly zoledronic acid for treatment of postmenopausal osteoporosis. N Engl J Med 356(18):1809-22

12. Black DM, Reid IR, Boonen S, et al (2012) The effect of 3 versus 6 years of zoledronic acid treatment of osteoporosis: a randomized extension to the HORIZON-Pivotal Fracture Trial (PFT). J Bone Miner Res 27(2):243-54

13. Lyles KW, Colón-Emeric CS, Magaziner JS et al (2007) Zoledronic acid in reducing clinical fracture and mortality after hip fracture. N Engl J Med 357(18):1799-809

14. Colón-Emeric CS, Mesenbrink P, Lyles KW et al (2010) Potential mediators of the mortality reduction with zoledronic acid after hip fracture. J Bone Miner Res 25(1):91-7 15. Brauer CA, Coca-Perraillon M, Cutler DM et al (2009) Incidence and mortality of hip fractures in the United States. JAMA 302(14):1573-9

16. Center JR, Bliuc D, Nguyen ND et al (2011) Osteoporosis medication and reduced mortality risk in elderly women and men. J Clin Endocrinol Metab 96(4):1006-14 17. Marx R (2003) Pamidronate (Aredia) and zoledronate (Zometa) induced avascular necrosis of the jaws: a growing epidemic. J Oral Maxillofac Surg 61(9):1238-9 18. Migliorati, CA (2003) Bisphosphonates and oral cavity avascular bone necrosis. J Clin Oncol 21(22):4253-4 
19. Ruggiero S, Mehrotra B, Rosenberg TJ et al (2004) Osteonecrosis of the jaws associated with the use of bisphosphonates: a review of 63 cases. J Oral Maxillofac Surg 62(5):527-34 20. Bagan JV, Murillo J, Jimenez Y et al (2005) Avascular jaw osteonecrosis in association with cancer chemotherapy. J Oral Pathol Med 34(2):120-3

21. Carter G, A. Goss, Doecke C (2005) Bisphosphonates and avascular necrosis of the jaw: a possible association. Med J Aust Apr 18;182:413-5

22. Purcell P, Boyd I (2005) Bisphosphonates and osteonecrosis of the jaw. Med J Aust 182(8):417-8

23. Woo SB, Hellstein JW, Kalmar JR (2006) Systematic review: bisphosphonates and osteonecrosis of the jaws. Ann Intern Med 144(10):753-61

24. Khosla S, Burr D, Cauley J et al (2007) American Society for Bone and Mineral Research. Bisphosphonate-associated osteonecrosis of the jaw: report of a task force of the American Society for Bone and Mineral Research. J Bone Miner Res 22(10):1479-91 25. Migliorati CA, Epstein JB, Abt E, Berenson JR (2011) Osteonecrosis of the jaw and bisphosphonates in cancer: a narrative review. Nat Rev Endocrinol 7(1):34-42

26. Pazianas M, Miller P, Blumentals WA et al (2007) A review of the literature on osteonecrosis of the jaw in patients with osteoporosis treated with oral bisphosphonates: prevalence, risk factors, and clinical characteristics. Clin Ther 29(8):1548-58 27. Sambrook PN, Chen JS, Simpson JM et al (2010) Impact of adverse news media on prescriptions for osteoporosis: effect on fractures and mortality. Med J Aust 193(3):154-6

28. Advisory Task Force on Bisphosphonate-Related Ostenonecrosis of the Jaws, American Association of Oral and Maxillofacial Surgeons. American Association of Oral and Maxillofacial Surgeons position paper on bisphosphonate-related osteonecrosis of the jaws. J Oral Maxillofac Surg 2007;65:369-76 
29. Ruggiero SL, Dodson TB, Assael LA et al (2009) American Association of Oral and Maxillofacial Surgeons position paper on bisphosphonate-related osteonecrosis of the jaw 2009 update. Task Force on Bisphosphonate-Related Osteonecrosis of the Jaws, American Association of Oral and Maxillofacial Surgeons. Aust Endod J 35(3):119-30

30. Rizzoli R, Burlet N, Cahall D et al (2008) Osteonecrosis of the jaw and bisphosphonate treatment for osteoporosis. Bone;42(5):841-7

31. Badros A, Weikel D, Salama A et al (2006) Osteonecrosis of the jaw in multiple myeloma patients: clinical features and risk factors. J Clin Oncol 24(6):945-52

32. Favia G, Pilolli GP, Maiorano E (2009) Histologic and histomorphometric features of bisphosphonate-related osteonecrosis of the jaws: an analysis of 31 cases with confocal laser scanning microscopy. Bone 45(3):406-13

33. Favia G, Pilolli GP, Maiorano E (2009) Osteonecrosis of the jaw correlated to bisphosphonate therapy in non-oncologic patients: clinicopathological features of 24 patients. J Rheumatol 36(12):2780-7

34. Borromeo GL, Tsao CE, Darby IB et al (2011) A review of the clinical implications of bisphosphonates in dentistry. Aust Dent J 56(1):2-9.

35. Hughes DE, Wright KR, Uy HL et al (1995) Bisphosphonates promote apoptosis in murine osteoclasts in vitro and in vivo. J Bone Miner Res 10(10):1478-87

36. Rogers MJ, Frith JC, Luckman SP et al (1999) Molecular mechanisms of action of bisphosphonates. Bone 24(5 Sppl):73S-79S

37. Russell RG, Rogers MJ, Frith JC et al (1999) The pharmacology of bisphosphonates and new insights into their mechanisms of action. J Bone Miner Res 14 Suppl 2:53-65 38. Santini D, Vincenzi B, Avvisati G et al (2002) Pamidronate induces modifications of circulating angiogenetic factors in cancer patients. Clin Cancer Res 8(5):1080-4 
39. Wood J, Bonjean K, Ruetz S et al (2002) Novel antiangiogenic effects of the bisphosphonate compound zoledronic acid. J Pharmacol Exp Ther 302(3):1055-61 40. Giraudo E, Inoue M, Hanahan D (2004) An amino-bisphosphonate targets MMP-9expressing macrophages and angiogenesis to impair cervical carcinogenesis. J Clin Invest 114(5):623-33

41. Teronen O, Laitinen M, Salo T et al (2000) Inhibition of matrix metalloproteinases by bisphosphonates may in part explain their effects in the treatment of multiple myeloma. Blood 96(12): 4006-7

42. Ferretti G, Fabi A, Carlini P et al (2005) Zoledronic-acid-induced circulating level modifications of angiogenic factors, metalloproteinases and proinflammatory cytokines in metastatic breast cancer patients. Oncology 69(1):35-43

43. Landesberg R, Woo V, Cremers S et al (2011) Potential pathophysiological mechanisms in osteonecrosis of the jaw. Ann NY Acad Sci 1218:62-79.

44. Gertz BJ, Holland SD, Kline et al (1993) Clinical pharmacology of alendronate sodium. Osteopor Int Suppl 3:S13-S16

45. Nancollas GH, Tang R, Phipps RJ et al (2006) Novel insights into actions of bisphosphonates on bone: differences in interactions with hydroxyapatite. Bone 8(5):617-27 46. Reid IR, Bolland MJ, Grey AB (2007) Is bisphosphonate-associated osteonecrosis of the jaw caused by soft tissue toxicity? Bone 41(3):318-20

47. Scheper MA, Badros A, Chaisuparat R et al (2009) Effect of zoledronic acid on oral fibroblasts and epithelial cells: a potential mechanism of bisphosphonate-associated osteonecrosis. Br J Haematol 144(5):667-76

48. Demerjian N, Bolla G, Spreux A (1999) Severe oral ulcerations induced by alendronate. Clin Rheumatol 18(4):349-50 
49. Lin JH (1996) Bisphosphonates: a review of their pharmacokinetic properties. Bone 18(2):75-85

50. Licata AA (2005) Discovery, clinical development, and therapeutic uses of bisphosphonates. Ann Pharmacother 39(4):668-77

51. Zahrowski JJ (2007) Comment on the American Association of Oral and Maxillofacial Surgeons statement on bisphosphonates. J Oral Maxillofac Surg 65(7):1440-1

52. Compston J (2011) Pathophysiology of atypical femoral fractures and osteonecrosis of the jaw. Osteoporos Int 22(12):2951-61

53. Sambrook P, Olver I, Goss A (2006) Bisphosphonates and osteonecrosis of the jaw. Aust Fam Physician 35(10):801-3

54. Marx RE, Sawatari Y, Fortin M, Broumand V (2005) Bisphosphonate-induced exposed bone (osteonecrosis/osteopetrosis) of the jaws: risk factors, recognition, prevention, and treatment. J Oral Maxillofac Surg 63(11):1567-75

55. Ruggiero SL (2011) Bisphosphonate-related osteonecrosis of the jaw: an overview. Ann N Y Acad Sci 1218:38-46

56. Taylor KH, Middlefell LS, Mizen KD (2010) Osteonecrosis of the jaws induced by antiRANK ligand therapy. Br J Oral Maxillofac Surg 48 (3):221-3

57. Aghaloo TL, Felsenfeld AL, Tetradis S (2010) Osteonecrosis of the jaw in a patient on Denosumab. J Oral Maxillofac Surg 68(5):959-63

58. Cummings SR, San Martin J, McClung MR et al (2009) FREEDOM Trial. Denosumab for prevention of fractures in postmenopausal women with osteoporosis. N Engl J Med 361(8):756-765

59. Papapoulos S, Chapurlat R, Libanati C et al (2012) Five years of denosumab exposure in women with postmenopausal osteoporosis: results from the first two years of the FREEDOM extension. J Bone Miner Res 27(3):694-701 
60. Biasotto M, Chiandussi S, Zacchigna S et al (2010) A novel animal model to study nonspontaneous bisphosphonates osteonecrosis of jaw. J Oral Pathol Med 39(5)390-6

61. Maahs MP, Azambuja AA, Campos MM et al (2011) Association between bisphosphonates and jaw osteonecrosis: a study in Wistar rats. Head Neck 33(2):199-207 62. Sonis ST, Watkins BA, Lyng GD et al (2009) Bony changes in the jaws of rats treated with zoledronic acid and dexamethasone before dental extractions mimic bisphosphonaterelated osteonecrosis in cancer patients. Oral Oncol 45(2):164-72

63. Aghaloo TL, Kang B, Sung EC et al (2011) Periodontal disease and bisphosphonates induce osteonecrosis of the jaws in the rat. J Bone Miner Res 26(8):1871-82

64. Allen MR (2009) Bisphosphonates and osteonecrosis of the jaw: moving from the bedside to the bench. Cells Tissues Organs 189 (1-4):289-94

65. Allen MR, Burr DB (2008) Mandible matrix necrosis in beagle dogs after 3 years of daily oral bisphosphonate treatment. J Oral Maxillofac Surg 66(5):987-94

66. Bi Y, Gao Y, Ehirchiou D et al (2010) Bisphosphonates cause osteonecrosis of the jawlike disease in mice. Am J Pathol 177(1):280-90

67. Kikuiri T, Kim I, Yamaza T et al (2010) Cell-based immunotherapy with mesenchymal stem cells cures bisphosphonate-related osteonecrosis of the jaw-like disease in mice. J Bone Miner Res 25(7):1668-79

68. Hellstein J, Marek C (2005) Bisphosphonate osteochemonecrosis (bis-phossy jaw): is this phossy jaw of the 21st century? J Oral Maxillofac Surg 63(5):682-9

69. Lugassy G, Shaham R, Nemets A et al (2004) Severe osteomyelitis of the jaw in longterm survivors of multiple myeloma: a new clinical entity. Am J Med 117(6):440-1 70. Durie B, Katz M, Crowley J (2005) Osteonecrosis of the jaw and bisphosphonates. N Engl J Med 353(1):99-102 
71. Vannuchi A, Ficarra G, Pogrel MA (2005) Osteonecrosis of the jaw associated with zoledronate therapy in a patient with multiple myeloma. Br J Haem 128:738.

72. Wang J, Goodger NM, et al (2003) Osteonecrosis of the jaws associated with cancer chemotherapy. J Oral Maxillofac Surg 61(7):1104-7

73. Hoff AO, Toth BB, Altundag K et al (2008) Frequency and risk factors associated with osteonecrosis of the jaw in cancer patients treated with intravenous bisphosphonates. $\mathrm{J}$ Bone Miner Res 23(6):826-36

74. Vahtsevanos K, Kyrgidis A, Verrou E et al (2009) Longitudinal cohort study of risk factors in cancer patients with bisphosphonate-related osteonecrosis of the jaw. J Clin Oncol 27(32):5356-62

75. Hess LM, Jeter JM, Benham-Hutchins M et al (2008) Factors associated with osteonecrosis of the jaw among bisphosphonate users. Am J Med 121(6):475-483 76. Tarassoff P, Csermak K (2003) Avascular necrosis of the jaws: risk factors in metastatic cancer patients. J Oral Maxillofac Surg 61(10):1238-9

77. Khamaisi M, Regev E, Yarom N et al (2007) Possible association between diabetes and bisphosphonate-related jaw osteonecrosis. J Clin Endocrinol Metab 92(3):1172-5

78. Migliorati C (2005) Bisphosphonate-associated oral osteonecrosis. Oral Surg Oral Med Oral Pathol Oral Radiol Endod 99(2):35

79. Yarom N, Yahalom R, Shoshani Y et al (2007) Osteonecrosis of the jaw induced by orally administered bisphosphonates: incidence, clinical features, predisposing factors and treatment outcome. Osteoporos Int 18(10):1363-70

80. Manfredi M, Merigo E, Guidotti R et al (2011) Bisphosphonate-related osteonecrosis of the jaws: a case series of 25 patients affected by osteoporosis. Int J Oral Maxillofac Surg 40(3):277-84

81. Sedghizadeh PP, Stanley K, Caligiuri M et al (2009) Oral bisphosphonate use and the 
prevalence of osteonecrosis of the jaw: an institutional inquiry. J Am Dent Assoc 140(1):61-6 82. Otto S, Abu-Id MH, Fedele S et al (2011) Osteoporosis and bisphosphonates-related osteonecrosis of the jaw: not just a sporadic coincidence-a multi-centre study. J Craniomaxillofac Surg 39(4):272-7

83. Bone HG, Hosking D, Devogelaer JP et al (2004) Ten years' experience with alendronate for osteoporosis in post-menopausal women. N Engl J Med 350(12): 1189-99

84. Reid IR (2009) Osteonecrosis of the jaw: who gets it, and why?

Bone 44(1):4-10

85. Solomon DH, Mercer E, Woo SB, Avorn J, Schneeweiss S, Treister N (2013)

Defining the epidemiology of bisphosphonate-associated osteonecrosis of the jaw: prior work and current challenges. Osteoporos Int. 24(1):237-44

86. Lapi F, Cipriani F, Caputi AP et al (2013) Bisphosphonates efficacy-safety tradeoff (BEST) study group. Assessing the risk of osteonecrosis of the jaw due to bisphosphonate therapy in the secondary prevention of osteoporotic fractures. Osteoporos Int 24(2):697-705

87. Edwards BJ, Hellstein JW, Jacobsen PL et al (2008) American Dental Association Council on Scientific Affairs Expert Panel on Bisphosphonate-Associated Osteonecrosis of the Jaw. Updated recommendations for managing the care of patients receiving oral bisphosphonate therapy: an advisory statement from the American Dental Association Council on Scientific Affairs. J Am Dent Assoc 139(12):1674-7

88. Grbic JT, Black DM, Lyles KW et al (2010) The incidence of osteonecrosis of the jaw in patients receiving 5 milligrams of zoledronic acid: data from the health outcomes and reduced incidence with zoledronic acid once yearly clinical trials program. J Am Dent Assoc 141(11):1365-70 
89. Papapoulos SE, Cremers SC (2007) Prolonged bisphosphonate release after treatment in children. N Engl J Med 356(10):1075-6

90. Hillner BE, Ingle JN, Chlebowski RT et al (2003) American Society of Clinical Oncology 2003 update on the role of bisphosphonates and bone health issues in women with breast cancer. J Clin Oncol 21(21):4042-57

91. Mavrokokki T, Cheng A, Stein B et al (2007) Nature and frequency of bisphosphonateassociated osteonecrosis of the jaws in Australia. J Oral Maxillofac Surg 65(3):415-23 92. Fellows JL, Rindal DB, Barasch A et al (2011) DPBRN Collaborative Group. ONJ in two dental practice-based research network regions. J Dent Res 90(4):433-8

93. Barasch A, Cunha-Cruz J, Curro FA et al (2011) Risk factors for osteonecrosis of the jaws: a case-control study from the CONDOR dental PBRN. J Dent Res 90(4):439-44 94. Lo JC, O'Ryan FS, Gordon NP et al (2010) Prevalence of osteonecrosis of the jaw in patients with oral bisphosphonate exposure. J Oral Maxillofac Surg 68(2):243-53 95 O'Ryan FS, Lo JC (2012) Bisphosphonate-related osteonecrosis of the jaw in patients with oral bisphosphonate exposure: clinical course and outcomes. J Oral Maxillofac Surg 70(8):1844-53.

96. Borromeo GL, Brand C, Clement JG et al (2011) Is bisphosphonate therapy for benign bone disease associated with impaired dental healing? A case-controlled study. BMC Musculoskelet Disord Apr 10;12:71

97. Baur DA, Weber JM, Collette DC, Dhaliwal H, Quereshy F (2012) Osteonecrosis of the jaws unrelated to bisphosphonate exposure: a series of 4 cases. J Oral Maxillofac

\section{$\underline{\text { Surg 70(12):2802-8 }}$}

98. Melo MD, Obeid G (2005) Osteonecrosis of the jaws in patients with a history of receiving bisphosphonate therapy: strategies for prevention and early recognition. J Am Dent Assoc 136(12):1675-81 
99. Migliorati CA, Casiglia J, Epstein J et al (2005) Managing the care of patients with bisphosphonate-associated osteonecrosis: an American Academy of Oral Medicine position paper. J Am Dent Assoc 136 (12):1658-68

100. Wooltorton E (2005) Patients receiving intravenous bisphosphonates should avoid invasive dental procedures. CMAJ 172(13):1684

101. Migliorati CA, Mattos K, Palazzolo MJ (2010) How patients' lack of knowledge about oral bisphosphonates can interfere with medical and dental care. J Am Dent Assoc 141(5):562-6

102. Ripamonti CI, Maniezzo M, Campa T et al (2009) Decreased occurrence of osteonecrosis of the jaw after implementation of dental preventive measures in solid tumour patients with bone metastases treated with bisphosphonates. The experience of the National Cancer Institute of Milan. Ann Oncol 20(1):137-45

103. Dimopoulos MA, Kastritis E, Bamia C et al (2009) Reduction of osteonecrosis of the jaw (ONJ) after implementation of preventive measures in patients with multiple myeloma treated with zoledronic acid. Ann Oncol 20(1):117-20

$\underline{\text { 104. McClung M, Harris ST, Miller PD et al (2013) Bisphosphonate therapy for }}$ osteoporosis: benefits, risks, and drug holiday. Am J Med 126(1):13-20

105. Mozzati M, Arata V, Gallesio G (2013)Tooth extraction in osteoporotic patients taking oral bisphosphonates. Osteoporos Int 24(5):1707-12.

105. Marx RE, Cillo JE Jr, Ulloa JJ (2007) Oral bisphosphonate-induced osteonecrosis: risk factors, prediction of risk using serum CTX testing, prevention, and treatment. J Oral Maxillofac Surg 65(12):2397-410

106. Cremers S, Farooki A. Biochemical markers of bone turnover in osteonecrosis of the jaw in patients with osteoporosis and advanced cancer involving the bone. Ann NY Acad Sci 2011;1218:80-7. 
107. Lee CY, Suzuki JB (2010) CTX biochemical marker of bone metabolism. Is it a reliable predictor of bisphosphonate-associated osteonecrosis of the jaws after surgery? Part II: a prospective clinical study. Implant Dent 19(1):29-38

108. American Society for Bone and Mineral Research Task Force on Osteonecrosis of the Jaw, Oral bisphosphonate-induced osteonecrosis: risk factors, prediction of risk using serum CTX testing, prevention, and treatment. J Oral Maxillofac Surg 2008;66:1320-1. 109. Greenberg M (2004) Intravenous bisphosphonates and osteonecrosis. Oral Surg Oral Med Oral Pathol Oral Radiol Endod 98(3): 259-60

110. Wutzl A, Pohl S, Sulzbacher I et al (2012) Factors influencing surgical treatment of bisphosphonate-related osteonecrosis of the jaws. Head Neck 34(2):194-200

111. Shimura K, Shimazaki C, Taniguchi K et al (2006) Hyperbaric oxygen in addition to antibiotic therapy is effective for bisphosphonate-induced osteonecrosis of the jaw in a patient with multiple myeloma. Int J Hematol 84(4):343-5

112. Freiberger JJ, Padilla-Burgos R, Chhoeu AH et al (2007) Hyperbaric oxygen treatment and bisphosphonate-induced osteonecrosis of the jaw: a case series. J Oral Maxillofac Surg 65(7):1321-7

113. Cheung A, Seeman E (2010) Teriparatide therapy for alendronate-associated osteonecrosis of the jaw. N Engl J Med 363(25):2473-4

114. Harper R, Fung E (2007) Resolution of bisphosphonate-associated osteonecrosis of the mandible: possible application for intermittent low-dose parathyroid hormone [rhPTH(1-34)]. J Oral Maxillofac Surg 65(3):573-80

115. Lau AN, Adachi JD (2009) Resolution of osteonecrosis of the jaw after teriparatide [recombinant human PTH-(1-34)] therapy. J Rheumatol 36(8):1835-7

116. Narongroeknawin P, Danila MI, Humphreys LG et al (2010) Bisphosphonate-associated 
osteonecrosis of the jaw, with healing after teriparatide: a review of the literature and a case report. Spec Care Dentist 30(2):77-82

117. Bashutski JD, Eber RM, Kinney JS et al (2010) Teriparatide and osseous regeneration in the oral cavity. N Engl J Med 363(25):2396-405

118. Junquera L, Gallego L (2008) Nonexposed bisphosphonate-related osteonecrosis of the jaws: another clinical variant? J Oral Maxillofac Surg 66(7):1516-7

119. Mawardi H, Treister N, Richardson P et al (2009) Sinus tracts-an early sign of bisphosphonate-associated osteonecrosis of the jaws? J Oral Maxillofac Surg 67(3):593-601 120. Fedele S, Porter SR, D'Aiuto F et al (2010) Nonexposed variant of bisphosphonate associated osteonecrosis of the jaw: a case series. Am J Med 123(11):1060-4

121. Khosla S, Bilezikian JP, Dempster DW et al (2012) Benefits and risks of

bisphosphonate therapy for osteoporosis. J Clin Endocrinol Metab 97(7):2272-82.

Table 1: Possible features of bisphosphonate related osteonecrosis of the jaw [24,31-34].

\author{
Clinical \\ dental pain \\ paraesthesia \\ halitosis \\ swelling \\ suppuration/oral discharge \\ ulceration \\ sinus tracks/fistulae \\ loosening of teeth \\ delayed/non-healing dental wounds
}

\title{
Radiological
}

Plain X-ray (orthopantomography, OPG): radiolucent or radio-opaque lesions with or without bony sequestra; useful for exclusion of malignancy 
CT: useful for defining extent of bony lesion and exclusion of malignancy

MRI: ischaemic region with non-enhancement following IV gadolinium

\section{Pathological}

mixed inflammatory cellular infiltrate

necrotic bone

presence of Actinomyces

reduced osteoclastic activity/new bone formation

reduced bone vascularity

Table 2: Bisphosphonate-related osteonecrosis of the jaw staging system proposed by the American Association of Oral and Maxillofacial Surgeons [29,55]

At risk category No apparent exposed/necrotic bone in patients who have been treated with either oral or IV bisphosphonates

Stage $0 \quad$ Non-specific clinical findings and symptoms such as jaw pain or osteosclerosis but no clinical evidence of exposed bone

Stage 1 Exposed/necrotic bone in patients who are asymptomatic and have no evidence of infection 
and erythema in the region of the exposed bone with or without purulent drainage

Stage 3

Exposed/necrotic bone in patients with pain, infection and one or more of the following: pathologic fracture, extra-oral fistula, or osteolysis extending to the inferior border or sinus floor

\section{Figure 1}

Osteonecrosis of the maxilla in a 51-year old female patient with Goodpasture's Syndrome who had undergone a cadaveric renal transplant 15 years earlier. She had been taking alendronate $70 \mathrm{mg}$ weekly for four years for glucocorticoid-induced osteoporosis and presented four months following multiple dental extractions with painful areas of non-healing exposed bone (arrow). There was no history of radiotherapy to the area. 


\section{Figure 2}

Osteo-radionecrosis in the (a) maxilla and (b) mandible of an 84-year old female patient mimicking ONJ (arrows). Initial referral was for assessment of osteonecrosis of the jaw secondary to BP use. Clinical assessment revealed a history of radiotherapy to the oro-facial region for previous malignancy. 

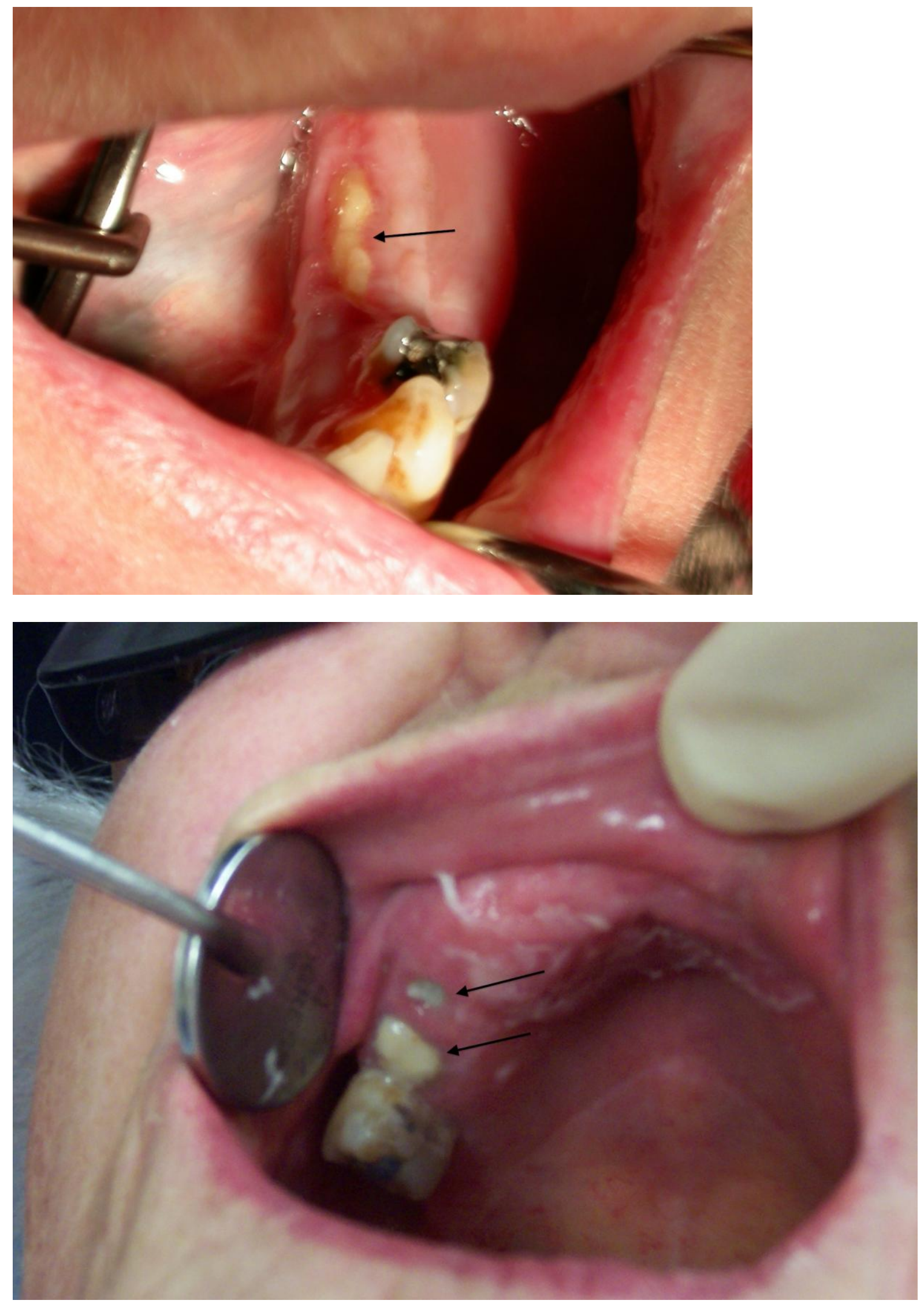


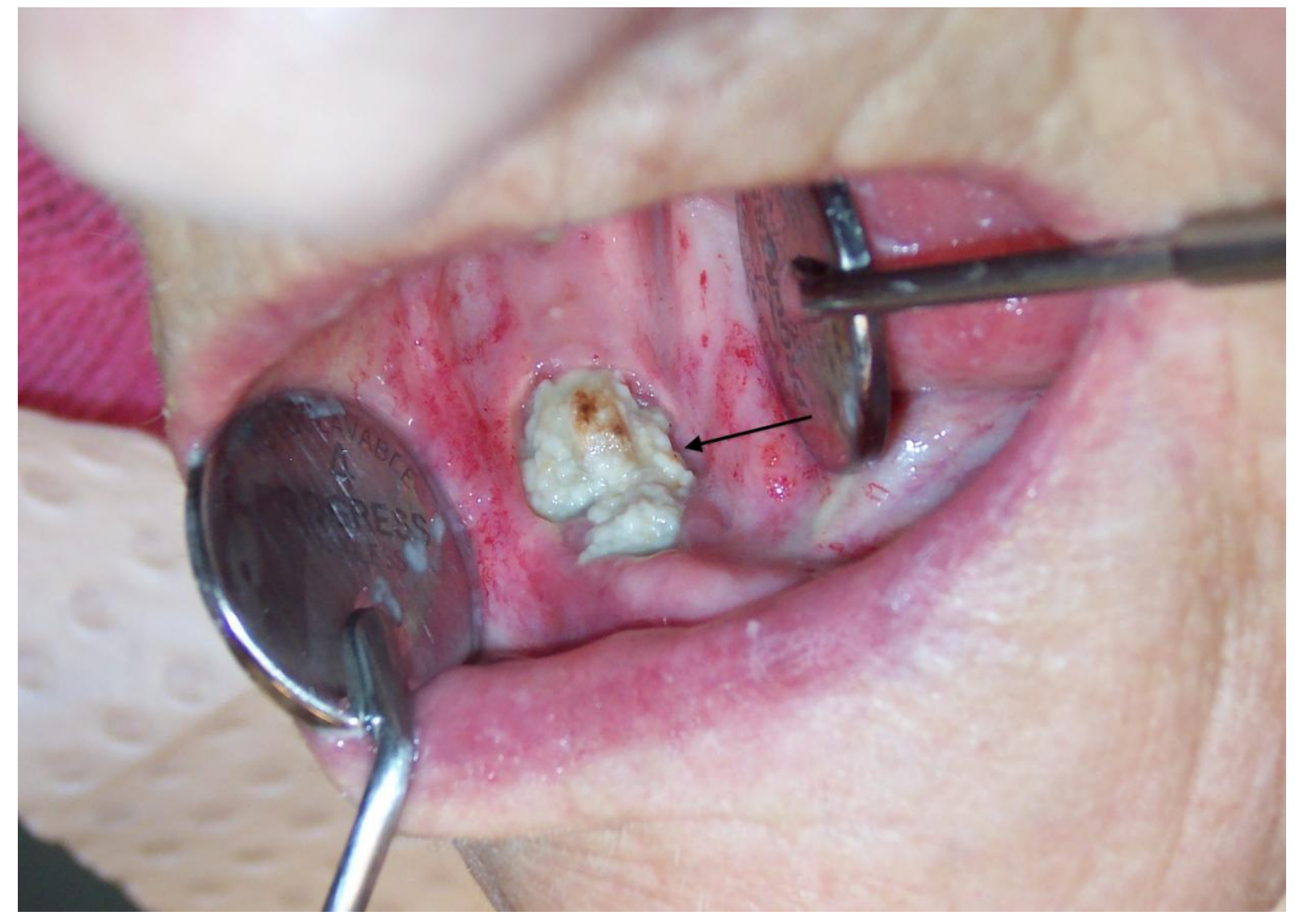

